\title{
Atomic Spectroscopy and the Photon Mass: Effects on the hydrogen ground-state
}

\author{
Miguel E. Rodriguez R. \\ Career of Pedagogy in Physics and Mathematics, \\ Faculty of Education, National University of Chimborazo, \\ Riobamba, Ecuador-060150. \\ ORCID: 0000-0001-8477-6612 (Miguel) \\ Maribel P. Pirela \\ Environmental Engineering, Faculty of Engineering, \\ National University of Chimborazo, Riobamba, Ecuador-060150. \\ ORCID: 0000-0002-9687-5471 (Maribel)
}

\begin{abstract}
The effect due to the photon mass is explored in the hydrogen atom. Specifically, the ground-state energy of the hydrogen atom within Proca's electrodynamics is considered. The resulting correction term is compared with the experimental precision of the ground-state energy of the hydrogen atom. From this, the limit of $3 \times 10^{-42} \mathrm{~g}$ on the photon mass is obtained.
\end{abstract}

\section{INTRODUCTION}

The photon is the particle that mediates the electromagnetic interaction. This particle appears as a result of the quantization of Maxwell's theory with the peculiarity that has no mass [12]. Furthermore, Proca [22] in 1936 introduced a relativistic generalization of the Maxwell equations which, after quantization, lead to a photon with mass [12]. Although the scenario of Maxwell has been extensively tested experimentally, Proca's scenario draws attention because it seems more intuitive that every particle in nature possesses mass. Consequently, the controversy that the photon possesses mass or not should be determined only by experimental verification. A similar controversy arose with the neutrino, which is the particle that mediates the strong nuclear interaction. This particle was considered massless like the photon, but recently, neutrino oscillation has been observed experimentally, which is only possible if the neutrino has mass [19][15]. Therefore, finding the photon mass (unlike neutrino mass) stands as an open and fundamental particle physics subject.

From a theoretical perspective, Proca's scenario involves the insertion of a mass term in the Lagrangian of quantum electrodynamics which breaks the gauge invariance, which implies that the theory can not be re-normalizable. However, this is not the case for the Proca Lagrangian, since it can be viewed as a gauge-fixed version of the Stückelberg Lagrangian [30], which restores gauge invariance [25]. In addition, a finite photon mass is perfectly compatible with the general principles of elementary particle physics. Of course, the enormous successes of quantum electrodynamics has led to an almost total acceptance that the photon has no mass. However, despite this acceptance, a substantial experimental effort has been made to determine, directly or indirectly, whether the photon mass is zero or nonzero.
From the experimental point of view, the difficulty of detecting the photon mass is that it can be extremely small. In this regard, the uncertainty principle in its energy-time version (i.e. $\Delta E \Delta t \geq \hbar / 2$ ) allows setting a final limit of the photon mass. Considering that $m_{\gamma}$ is the mass of the photon and $\Delta t=10^{10}$ years (age of the universe), then $m_{\gamma} \approx \hbar /(\Delta t) c^{2}=10^{-66} g$. As $m_{\gamma}$ can be extremely small, the best we can do is placing even tighter limits on its size.

The procedure for establishing a limit on $m_{\gamma}$ depends on the studied phenomena, which can be classified into two types, namely, type 1: long-range and/or low experimental precision phenomena, or type 2: short-range and high experimental precision phenomena. Among the phenomena of type 1 are the measurements of deflection of the magnetic dipole field of the earth made by Fischbach et al. [6] and Goldhaber and Nieto [10]. A more extensive list of methods of type 1 can be found in the revision of Tu et. al. [33] and Goldhaber and Nieto [9][11]. Among the tests of type 2 phenomena, we have the highly accurate test of the Coulomb law by William et al. [34] and the cryogenic experiment by Ryan et al. [26]. As in the phenomena of type 2 precision is critical, then it is reasonable to explore quantum phenomena in which this requirement is present. Boulware and Deser were the first to explore quantum scenarios by studying the quantum AharonovBohm effect with Proca's theory [2]. Subsequently, the same approach was extended to other quantum effects of the same type as the Aharonov-Casher effect [7], the electron-positron effect [29] and Tackchuk effect [32]. The effect of type AB neutron proposed by Sangster et. al. [27] was studied by M. Rodriguez [23]. To broaden the discussion on the photon mass in quantum scenarios, one can consult the paper by Spavieri et. al. [28].

Recently, Caccavano and Leung [3], have explored the effect of the photon mass in atomic spectrometry, specifically, they 
have recalculated the hyperfine interaction using the theory of Proca and they have obtained a correction term for line $21 \mathrm{~cm}$ hydrogen. However, Caccavano and Leung [3] do not set a limit on $m_{\gamma}$ because the correction term is too small to be detected with current spectroscopic technology. Following this approach, in this work the effect of the mass of the photon on ground-state energy of the hydrogen atom is explored and an experimental limit on $m_{\gamma}$ is obtained. Our work is organized as follows: in section II Proca equations are presented and from these the electrostatic potential for a charged particle is derived. In section III the Schrödinger equation with the potential found in section II (Yukawa potential) is presented and some approximate analytical methods are mentioned to find the ground-sate energy. In Section VI a limit on $m_{\gamma}$ is established. Finally, in section V the results are discussed.

\section{PROCA'S EQUATIONS AND YUKAWA POTENTIAL}

\section{Proca's equations}

The set of equations of the electromagnetic field leading to massive photon known as Proca equations, which in SI units are as follows:

$$
\begin{gathered}
\nabla \cdot \mathbf{E}=\frac{\rho}{\varepsilon_{0}}-\mu_{\gamma}^{2} \phi \\
\nabla \times \mathbf{E}=-\frac{\partial \mathbf{B}}{\partial t}, \\
\nabla \cdot \mathbf{B}=0, \\
\nabla \times \mathbf{B}=\mu_{0} \mathbf{J}+\mu_{0} \varepsilon_{0} \frac{\partial \mathbf{E}}{\partial t}-\mu_{\gamma}^{2} \mathbf{A}
\end{gathered}
$$

where $\mathbf{E}$ is the electric field, $\mathbf{B}$ the magnetic field, $\rho$ the charge density, $\mathbf{J}$ the current density, $\phi$ the scalar potential, $\mathbf{A}$ the vector potential, $\mu_{0} \mathrm{y} \varepsilon_{0}$ the permittivity and permeability of free space and $\mu_{\gamma}$ a characteristic length of the related theory (through quantization [12])) with the photon mass, i.e.:

$$
m_{\gamma}=\frac{\mu_{\gamma} \hbar}{c}
$$

Additionally, the potentials maintain their standard form,

$$
\mathbf{B}=\nabla \times \mathbf{A},
$$

and

$$
\mathbf{E}=-\nabla \phi-\frac{\partial \mathbf{A}}{\partial t}
$$

with the condition of Lorentz

$$
\nabla \cdot \mathbf{A}+\frac{1}{c^{2}} \frac{\partial \phi}{\partial t}=0
$$

which it is necessary for the validity of the conservation of charge. Should be noted that due to parameter $\mu_{\gamma}$ present in equations (1) and (4) $\mathbf{E}$ and $\mathbf{B}$ fields acquire an exponential attenuation, additional natural dependence on the distance, hence Proca's electrodynamics is also known as finite-range electrodynamics .

\section{Yukawa potential}

To obtain the equation of scalar potential in the Proca's theory (7) is replaced (as is usual in Maxwell's theory) in Gauss's law modified (1), provided $\partial_{t} \mathbf{A}=0$, this gives,

$$
\left(\nabla^{2}-\mu_{\gamma}^{2}\right) \phi(r)=-\frac{\rho(r)}{\varepsilon_{0}},
$$

which has the form of an inhomogeneous modified Helmholtz equation. For a point particle $\rho(r)=q \delta(r)$ and (9) becomes as follows:

$$
\left(\nabla^{2}-\mu_{\gamma}^{2}\right) \phi(r)=-\frac{q}{\varepsilon_{0}} \delta(r)
$$

The solution is [1]:

$$
\phi(r)=\frac{q}{4 \pi \varepsilon_{0}} \frac{e^{-\mu_{\gamma} r}}{r} .
$$

This potential is known in the literature with several names depending on the application area. In nuclear physics, it is known as Yukawa potential and is used to model the strong interaction between nucleon-nucleon due to the exchange of a meson [35]. In solid-state physics it is called Fermi-Dirac potential [18] and in physics of plasmas it is known as DebeyHuckle potential [16][21].

\section{HYDROGEN ATOM WITH YUKAWA POTENTIAL}

The Schrödinger equation independent of time for the hydrogen atom with the Yukawa potential (11) is as follows:

$$
\left(-\frac{\hbar^{2}}{2 m} \nabla^{2}+\frac{e^{2}}{4 \pi \varepsilon_{0}} \frac{e^{-\mu_{\gamma} r}}{r}\right) \psi(r, \theta, \varphi)=E \psi(r, \theta, \varphi),
$$

where spherical coordinates have been adopted because of the symmetry of the potential. Applying separation of variables 
$\psi(r, \theta, \varphi)=\frac{R(r)}{r} Y(\theta, \varphi)[13]$ radial Schrödinger equation is obtained, that is to say:

$$
\left[\frac{d^{2}}{d r^{2}}+2 m\left[\frac{E}{\hbar^{2}}+\frac{e^{2}}{4 \pi \epsilon_{0} \hbar^{2}} \frac{e^{-\mu_{\gamma} r}}{r}\right]-\frac{l(l+1)}{r^{2}}\right] R_{n l}=0
$$

this equation does not allow exact solutions due to the presence of the exponential term in the potential. Consequently, many approximate methods have been applied for its solution. For example, it has been used perturbation theory [31], variational method [4][24] and group theory [8]. Hamzavi et al recently [14] obtained by the parametric generalized method of Nikiforov-Uvarov [20], approximate analytical solutions of the Schrödinger radial equation. With this method Hamzavi et al. [14] obtained the energy eigenvalues and the corresponding eigenfunctions approximate equation (13) in closed form. To make the analysis of the photon mass in the context of the hydrogen atom here the results obtained by Hamzavi et. al. [14] will be used. Specifically, the expression of the eigenvalues of energy (equation (19) in [14]) will be taken, i.e.:

$$
E_{n, l}=-\frac{\hbar^{2}}{2 m}\left[\frac{m \frac{e^{2}}{4 \pi \epsilon_{0} \hbar^{2}}-\mu_{\gamma} n^{2}}{n}\right]^{2}
$$

where $n=j_{\max }+l+1, j_{\max }=0,1,2 \ldots$ and $l=$ $0,1,2 \ldots[13]$. Ground state energy is achieved when $n=1$. In this case the expression (14) is reduced as follows:

$$
E_{1,0}=\left(E_{1,0}\right)_{Y}=-\frac{\hbar^{2}}{2 m}\left[m \frac{e^{2}}{4 \pi \epsilon_{0} \hbar^{2}}-\mu_{\gamma}\right]^{2}
$$

which it has been added the subscript $Y$ to identify which is the power base of the hydrogen atom with Yukawa potential state. Note that in the limit when $\mu_{\gamma} \rightarrow 0$ the expression (15) is reduced to the ground-state energy of the hydrogen atom with Coulomb potential, i.e.,

$$
\left(E_{1,0}\right)_{C}=\lim _{\mu_{\gamma} \rightarrow 0}\left(E_{1,0}\right)_{Y}=-\frac{m}{2 \hbar^{2}}\left(\frac{e^{2}}{4 \pi \epsilon_{0}}\right)^{2} .
$$

\section{IV: LIMIT ON $\mu_{\gamma}$ AND $M_{\gamma}$}

The standard procedure adopted to set a limit on $m_{\gamma}$ is set a limit on $\mu_{\gamma}$ first. This is done by comparing the additional contributions derived from the equations of Proca with the experimental precision of the studied phenomenon. This is useful for writing (15) as:

$$
\left(E_{1,0}\right)_{Y}=-\left[\left(E_{1,0}\right)_{C}+\frac{\left(\mu_{\gamma} \hbar\right)^{2}}{2 m}-\alpha c\left(\mu_{\gamma} \hbar\right)\right],
$$

where $E_{1, C}$ is defined by (16), $\alpha$ is the fine-structure constant and $c$ is the speed of light. Note that in (17) the last two terms on the left side satisfy the relation:

$$
\frac{\left(\mu_{\gamma} \hbar\right)^{2}}{2 m} \ll \alpha c\left(\mu_{\gamma} \hbar\right)
$$

This is because $\mu_{\gamma} \ll 1$ and $\frac{\hbar^{2}}{2 m} \ll \alpha c \hbar$. Therefore, the first order expression (17) is reduced to:

$$
\left(E_{1,0}\right)_{Y} \simeq-\left[\left(E_{1,0}\right)_{C}-\Delta E\left(\mu_{\gamma}\right)\right]
$$

where,

$$
\Delta E\left(\mu_{\gamma}\right)=\alpha c \mu_{\gamma} \hbar
$$

The expression (19) is the correction to be compared with the experimental error. This expression, also, can be obtanied like a perturbation when the Yukawa potential is expanded in Maclaurin to $\mu_{\gamma}$, because $\mu_{\gamma} \ll 1$. According to the NIST Atomic Spectra Database [17] the reported ground-energy state of hydrogen is:

$$
\left(E_{1,0}\right)_{C}=-13,598434005136(12) \mathrm{eV}
$$

Therefore, the experimental error of the ground-state of the hydrogen atom is $\left(\Delta E_{1,0}\right)_{C}=1,2 \times 10^{-11} \mathrm{eV}$.

So,

$$
\Delta E\left(\mu_{\gamma}\right)=\alpha c \mu_{\gamma} \hbar \leq\left(\Delta E_{1,0}\right)_{C} .
$$

With this the limit on $\mu_{\gamma}$ is obtained,

$$
\mu_{\gamma} \leq \frac{\left(\Delta E_{1,0}\right)_{C}}{\alpha c \hbar}=8,4 \times 10^{-3} m^{-1}
$$

Finally, substituting (21) in (5) the limit sought is obtained:

$$
m_{\gamma}=\mu_{\gamma} \frac{\hbar}{c} \leq \frac{\left(\Delta E_{1,0}\right)_{C}}{\alpha c^{2}}=3 \times 10^{-42} \mathrm{~g} .
$$

Notice that here we have not considered other interactions such as spin-orbit or hyperfine-structure, this is because the spinorbit is of the order of $\alpha^{4}$ and the hyperfine-structure is of the order of $\left(m / m_{p}\right) \alpha^{4} m c^{2}$ which are terms much smaller than (19). In fact, in the work of Caccavano and Leung [3] it is reported that the correction due to the hyperfine structure is of the order of $\sim\left(m / m_{p}\right) \alpha^{4} m c^{2} a_{0} \mu_{\gamma}^{2}$, with $a_{0}$ is the Bohr radius, $m$ is the electron mass and $m_{p}$ is the proton mass. Consequently, the greatest order correction obtained due to $\mu_{\gamma}$ is the expression (19) which is proportional to $\alpha$.

\section{v. CONCLUSIONS}

We obtained a limit on the mass of the photon through groundstate energy of the hydrogen atom. The limit found is $3 \times$ $10^{-42} \mathrm{~g}$ which is 7 orders of magnitude smaller the value 
that can be derived from the work of Caccavano and Leung [3]. Additionally, the scenario explored here differs from other methods that have been studied in the sense that the correction also depends on $\mu_{\gamma}$ in equation (17) and not only square product of the mass of the photon and the dimension of the phenomenon studied, i.e. $\left(\mu_{\gamma} D\right)^{2}$ as it expresses a theorem Goldghaber and Nieto [9]. This is because the interaction studied does not depend on the dimension of space. Furthermore, an experimental limit on the coefficient of shielding Yukawa potential is found (equation 21), which is often used to compute bound-state normalizations and energy levels of neutral atoms [5]. In fact, in the work Hamzavi et al. [14] values of $\mu_{\gamma}$ are imposed in order to obtain the different energy levels. For example, in the case of groundstate hydrogen Hamzavi et al. [14] reports that $\mu_{\gamma}=2 \times$ $10^{-14} \mathrm{~m}^{-1}$ that would imply a photon mass $m_{\gamma}=7 \times$ $10^{-56} \mathrm{~g}$. The point is that for a mass of this value the precision ground-state energy should be $\left(\Delta E_{1,0}\right)_{C}=2,7 \times 10^{-23} \mathrm{eV}$ which is 12 orders of magnitude smaller than the precision currently reported [17]. Finally, it is important to mention that the limit found represents an improvement by an order of magnitude with respect to the limits obtained with the quantum effects by Tkachuk $\left(m_{\gamma} \leq 2.5 \times 10^{-41} \mathrm{~g}\right)$ [32] and Sangster $\left(m_{\gamma} \leq 1.1 \times 10^{-41} \mathrm{~g}\right)$ [23], confirming once more that quantum approaches are a competitive way to find the limit of the photon mass.

\section{REFERENCES}

[1] G. B. Arfken and H Weber. Mathematical Methods For Physicists. Academic Press, 1 edition, 2004.

[2] David G. Boulware and S. Deser. Aharonov-bohm effect and the mass of the photon. Phys. Rev. Lett., 63(21):2319-2321, Nov 1989.

[3] A. Caccavano and P. T. Leung. Atomic spectroscopy and the photon mass: Effects on the $21 \mathrm{~cm}$ radiation. Physics Letters A, 377(39):2777 - 2779, 2013.

[4] M.a Demiralp, N.A.b Baykara, and H.b Taşeli. A basis set comparison in a variational scheme for the yukawa potential. Journal of Mathematical Chemistry, 11(1):311-323, 1992.

[5] R. Dutt and Y.P. Varshni. An analytic approximation for the energy levels of neutral atoms. Zeitschrift für Physik A Atoms and Nuclei, 313(3):143-145, 1983.

[6] E. Fischbach, H. Kloor, R. A. Langel, A. T. Y. Lui, and M. Peredo. New geomagnetic limits on the photon mass and on long-range forces coexisting with electromagnetism. Phys. Rev. Lett., 73(4):514-517, Jul 1994.

[7] Christopher Fuchs. Aharonov-casher effect in massive-photon electrodynamics. Phys. Rev. D, 42(8):2940-2942, Oct 1990.

[8] J.P.a Gazeau and A.a b Maquet. Bound states in a yukawa potential: A sturmian group-theoretical approach. Physical Review A, 20(3):727-739, 1979.

[9] A S Goldhaber and M M Nieto. Terrestrial and extraterrestrial limits on the photon mass. Rev. Mod. Phys., 43(3):277-296, Jul 1971.

[10] Alfred S. Goldhaber and Michael Martin Nieto. New geomagnetic limit on the mass of the photon. Phys. Rev. Lett., 21(8):567-569, Aug 1968.

[11] Alfred Scharff Goldhaber and Michael Martin Nieto. Photon and graviton mass limits. Rev. Mod. Phys., 82:939-979, Mar 2010.
[12] W. Greiner and J. Reinhardt. Field Quantization. SpringerVerlag, 1 edition, 1996.

[13] David J. Griffiths. Introduction to Quantum Mechanics. 2nd Edition. Pearson Prentice Hall, 2nd edition, 2004.

[14] M. Hamzavi, M. Movahedi, K.-E. Thylwe, and A.A. Rajabi. Approximate analytical solution of the yukawa potential with arbitrary angular momenta. Chin. Phys. Lett., 29(8):080302-1, 2012.

[15] T. Kajita. Nobel lecture: Discovery of atmospheric neutrino oscillations. Reviews of Modern Physics, 88(3), 2016.

[16] S. Kar and Y. K. Ho. Bound states and resonance states of the plasma-embedded td $\mu$ and $\mathrm{dd} \mu$ molecular ions. Eur. Phys. J. D, 48, 072008.

[17] A. Kramida, Yu. Ralchenko, J. Reader, and and NIST ASD Team. NIST Atomic Spectra Database (ver. 5.7.1), [Online]. Available: https://physics.nist.gov/asd [2020, April 30]. National Institute of Standards and Technology, Gaithersburg, MD., 2019.

[18] J. B. Kriegar. Electron shielding in heavily doped semiconductors. Physical Review (Series I), 178, 21969.

[19] A.B. McDonald. Nobel lecture: The sudbury neutrino observatory: Observation of flavor change for solar neutrinos. Reviews of Modern Physics, 88(3), 2016.

[20] Uvarov V. Nikiforov A. F. Special Functions of Mathematical Physics: A Unified Introduction with Applications. Birkhäuser, 1 edition, 1988.

[21] A. Poszwa. Dirac electron in the two-dimensional debye-yukawa potential. Physica Scripta, 89(6), 2014.

[22] A Proca. Sur la théorie du positron. Acad. Sci., Paris, Comptes Rendus, 8:202, 1938.

[23] M. Rodriguez. Masa del fotón por medio de un condensador de placas paralelas. Rev. Mex. Fis., 55(5):373-377, 2009.

[24] K.M.a Roussel and R.F.b c O'Connell. Variational solution of schrödinger's equation for the static screened coulomb potential. Physical Review A, 9(1):52-56, 1974.

[25] H. Ruegg and M. Ruiz-Altaba. The stueckelberg field. Int. J. Mod. Phys. A, 19(20):3265-3347, 2004.

[26] John J. Ryan, Frank Accetta, and Robert H. Austin. Cryogenic photon-mass experiment. Phys. Rev. D, 32(3):802-805, Aug 1985.

[27] Karin Sangster, E. A. Hinds, Stephen M. Barnett, and Erling Riis. Measurement of the aharonov-casher phase in an atomic system. Phys. Rev. Lett., 71(22):3641-3644, Nov 1993.

[28] G. Spavieri, J. Quintero, T. G. Gillies, and M. Rodríguez. A survey of existing and proposed classical and quantum approaches to the photon mass. Eur. Phys. J. D, 61(3):531-550, 2011.

[29] G. Spavieri and M. Rodriguez. Photon mass and quantum effects of the aharonov-bohm type. Phys. Rev. A, 75(5):052113, Dec 2007.

[30] E. C. G. Stueckelberg. Théorie de la radiation de photons de masse arbitrairement petite. Helv. Phys. Acta, 30:209-215, 1957.

[31] H. Taşeli and M. Demiralp. Convergent perturbation studies in screened coulomb potential systems: analytic evaluations up to third order for the yukawa case. Theoretica Chimica Acta, 71(4):315-325, 1987.

[32] V. M. Tkachuk. Quantum topological phase of an electric dipole circulating around a ferromagnetic wire. Phys. Rev. A, 62(5):052112, Oct 2000.

[33] Liang-Cheng Tu, Jun Luo, and George T Gillies. The mass of the photon. Rep. Prog. Phys., 68(1):77-130, Jan 2005.

[34] E. R. Williams, J. E. Faller, and H. A. Hill. New experimental test of coulomb's law: A laboratory upper limit on the photon rest mass. Phys. Rev. Lett., 26(12):721-724, Mar 1971.

[35] H. Yukawa. On the interaction of elementary particles. Proc. Phys. Math. Soc. Japan, 17:48, 1935. 